\title{
Datenverarbeitung im Recht
}

Archiv tür die gesamte Wissenschaft der Rechtsinformatik, der Rechtskybernetik und der Datenverarbeitung in Recht und Verwaltung.

Zitierweise: DVR

Herausgeber:

Dr. jur. Bernt Bühnemann. Wissenschaftlicher Oberrat an der Universität Hamburg Professor Dr. jur. Dr. rer. nat. Herbert Fiedler, Universität Bonn/Gesellschaft für Mathematik und Datenverarbeitung, Birlinghoven

D. jur. Hermann Heussner, Vorsitzender Richter am Bundessozialgericht, Kassel, Lehrbeauftragter an der Universität Gießen

Professor Dr. jur. Dr. phil. Adalbert Podlech, Technische Hochschule Darmstadt

Professor Dr. jur. Spiros Simitis, Universität Frankfurt a. M.

Professor Dr. jur Wilhelm Steinmüller, Universität Regensburg

Dr. jur. Sigmar Uhlig, Regierungsdirektor im Bundesministerium der Justiz, Bonn (Geschäftsführender Herausgeber)

Beratende Herausgeber und ständige Mitarbeiter:

Dr. Hélène Bauer Bernet, Service juridique commission C. E., Brüssel - Pierre Catala, Professeur à la Faculté de Droit de Paris, Directeur de I'Institut de Rechercres et d'Etudes pour le Traitement de I'Information Juridique de Montpellier Prof. Dr. jur. Wilhelm Dodenhoff, Vors. Richter am Bundesverwaltungsgericht, Berlin - Dr. Aviezri S. Fraenkel, Department of Applied Mathematics, The Weizman Ins:itute of Science, Rehovot - Prof. Dr. jur. Dr. phil. Klaus J. Hopt, M. C. J., Universtat Tübingen - Prof. Ejan Mackaay. Director of the Jurimetrics Research Group, Université de Montréal - mr. Jan Th. M. Palstra, Nederlandse Economische Hogeschool, Rotterdam - Professor Dr. Jürgen Rödig f, Universität Gießen - DirekIor Stb. Dr. jur. Otto Simmler, Administrative Bibliothek und Österreichische Rechtsdokumentation im Bundeskanzleramt, Wien - Frofessor Dr. Lovro Sturm, Irstitute of Public Administration, University in Ljubljana - Professor Dr. jur. Dieter Suhr, Freie Universitat Berin - Protessor Colin F. Tapper, Magdalen Co:!rge, Oxford -- lic. jur. Bernhard Vischer, UNIDATA AG, Zürich - Dr. Vladimir Vrecion, Juristische Fakuitat der Karls-Universität in Prag.

Geschäftsführender Herausgeber:

Dr. Sigmar Uhlig, D-53 Bonn-Tannenbusch, An der Düne 13,

Telefon 022 21/661378 (privat); $02221 / 581$ oder 584827 (dienstlich)

Fedaktioneller Mitarbeiter:

Oieter Hebebrand, D-3501 Niestetal. Fliederweg 1, Telefon 0561 / 524631 (privat); $0561 / 307363$ (Bundessozialgericht)

Manuskripte, redaktionelle Anfragen und Besprechungsexemplare werden an den Geschäftsführenden Herausgeber erbeten, geschäftliche Mitteilungen an den Verlag. Für unverlangt eingesandte Manuskripte wird keine Gewähr geleistet.

Die Beiträge werden nur unter der Voraussetzung aufgenommen, daß der Verfasser denselben Gegenstand nicht gleichzeitig in einer anderen Zeitschrift behandelt. Mit der Cberlassung des Manuskripts überträgt der Verfasser dem Verlag auf die Dauer des urheberrechtlichen Schutzes auch das Recht, die Herstellung von photomechanischen Vervielfältigungen in gewerblichen Unternehmen zum innerbetrieblichen Gebrauch zu genehmigen, wenn auf jedes Photokopieblatt eine Wertmarke der Inkassostelle des Börsenveroins des Deutschen Buchhandels in 6 Frankfurt a. M., Grober Hirschgraben 17/19 nach dem leweils geltenden Tarif aufgeklebt wird. 